\title{
Data Mining for Monitoring and Managing Systems and Networks
}

\author{
Antonio Liotta $\cdot$ Giuseppe Di Fatta
}

Received: 29 January 2014/ Accepted: 13 February 2014/Published online: 20 February 2014

(C) Springer Science+Business Media New York 2014

Modern communication networks have reached a level of scale, dynamics and complexity that demands for sophisticated management and control, driving many researchers towards the investigation of computational intelligence in the particular context of networks [1,2]. This is a difficult proposition, which requires a crossdisciplinary approach, pulling together efforts from a variety of research communities. During the last few decades we have seen substantial advances in topical areas such as statistical analysis, autonomic computing, machine learning and data mining, to master the significance of massive data sets. However, the use of such techniques in the context of communication networks has been investigated only recently.

To capture relevant work-in-progress, in 2011 we started the IEEE ICDM Workshop on Data Mining in Networks (now at its third edition, http://damnet. reading.ac.uk/), to facilitate a rich exchange of ideas among computer scientists, network experts and life scientists, who share a common interest in extracting models and information from any kind of complex networks (both natural and manmade). Encouraged by the outcomes of DaMNet we decided to create this Journal Special Issue, which has a more specific focus on the application of data mining to communication networks and addresses open issues in network control and management.

Overall we received thirteen submissions, covering a wide range of topics, theories, applications, analytical models and simulations. We started a rigorous peer-review process that was not free from controversies and conflicting reviews. We discovered how difficult it is to evaluate manuscripts that sit at the intersection

\footnotetext{
A. Liotta $(\square)$

Eindhoven University of Technology, Eindhoven, The Netherlands

e-mail: a.liotta@tue.nl

G. Di Fatta

University of Reading, Reading, UK

e-mail: g.difatta@reading.ac.uk
} 
of multiple disciplines. In fact, in quite a few cases we have had to allocate up to five reviewers per manuscript, to cover all related areas and find the right balance between divergent opinions. In the end, we selected five articles that, we hope, will feed your curiosity and, at the same time, provide a snapshot of the current state-ofplay.

We start this special issue with a survey on the "Application of Bayesian Networks for Autonomic Network Management", by A. Bashar, G. Parr, S. McClean, B. Scotney, and D Nauck. The authors describe the key challenges in modern network management and pinpoint the desired features to motivate the need for new learning-based approaches. Finally, they give practical examples in the context of admission control and traffic engineering.

The paper "A Novel Framework of Automated RRM for LTE SON", by M.I. Tiwana, brings the value of data mining directly into LTE (the Long Term Evolution telecommunication network), particularly for self-organization, selfhealing and traffic efficiency. The author shows how to derive the functional relationship between the Key Performance Indicators and the Radio Resource Management parameters and, then, use such prediction models to automate network monitoring and load-balance the traffic.

The paper "Topology-aware Correlated Network Anomaly Event Detection and Diagnosis”, by P. Calyam, M. Dhanapalan, M. Sridharan, A. Krishnamurthy, and R. Ramnath, adopts reinforcement learning principles to tackle the hard problem of anomaly detection in multi-domain networks. Their scheme is demonstrated in the context of network monitoring to detect and diagnose network-wide anomaly events.

The paper "Network Load Prediction based on Big Data and the Utilization of Self-organizing Maps", by A. Bantouna, G. Poulios, K. Tsagkaris, and P. Demestichas, employs unsupervised learning principles to detect traffic patterns and make predictions about the network load. This work suggests avenues to enhance network management procedures through a clever use of disparate data that is readily available to the network operator, while at the same time reducing the amount of historical data that needs to be stored.

The paper "Cloning SIM Cards Usability Reduction in Mobile Networks", by M.A. Al-Fayoumi and N.F. Shilbayeh, places the focus on security issues in the context of mobile networks. The authors introduce a probabilistic model to facilitate the detection of cloned SIM cards, to better address fraud in cellular networks.

We hope you will enjoy reading this special issue as much as we enjoyed putting it together and that you find these articles inspiring and useful. Our utmost gratitude goes to the anonymous reviewers for offering their time and expertise during two reviewing rounds. Special appreciation goes to the editor in chief, Deep Medhi, and the Springer staff for their valuable suggestions and help.

\section{References}

1. Liotta, A.: The cognitive NET is coming. IEEE Spectr. 50(8), 26-31 (2013). doi:10.1109/MSPEC. 2013.6565557 
2. Liotta, A., Exarchakos, G.: Networks for Pervasive Services: Six Ways to Upgrade the Internet. Springer, Berlin (2011). doi:10.1007/978-94-007-1473-1

Antonio Liotta holds the Chair of Communication Network Protocols at the Eindhoven University of Technology (The Netherlands), where he leads the Smart Networks team (http://bit.ly/autonomic networks) since 2008. Antonio is a Fellow of the UK Higher Education Academy and serves the Peer Review College of the UK Engineering and Physical Sciences Research Council. He is editor-in-chief of the book series Internet of Things: Technology, Communications and Computing (Springer), associate editor of the Journal of Network and System Management (Springer), and serves the editorial board of five more journals. During the last decade, he has investigated topical issues in the area of computer and multimedia networking and is currently studying cognitive systems in the context of optical, wireless and sensor networks. He has five patents and over 170 publications to his credit, and is the author of "Networks for Pervasive Services: six ways to upgrade the Internet" (Springer, $2011 \mathrm{http} / / / \mathrm{bit} . l \mathrm{ly} /$ pervasive-networks). His work on smart networks has been featured by IEEE Spectrum in "The cognitive Net is coming" (August 2013).

Giuseppe Di Fatta is an Associate Professor of Computer Science at the University of Reading, Reading, UK, where he has been since 2006. He is the Director of the MSc in Advanced Computer Science. In 1999, he was a research fellow at the International Computer Science Institute (ICSI), Berkeley, CA, USA. From 2000 to 2004, he was with the High-Performance Computing and Networking Institute of the National Research Council, Italy. From 2004 to 2006, he was with the University of Konstanz, Konstanz, Germany. He serves in the editorial board of the Elsevier Journal of Network and Computer Applications (JNCA). His research interests include data mining, scalable algorithms, distributed and parallel computing, and multidisciplinary applications. He has published over 60 articles in peer-reviewed conferences and journals. He has organised and chaired international workshops and conferences in data mining, distributed systems and computer networks, and is the co-founder of the IEEE ICDM Workshop on Data Mining in Networks (DaMNet). 